\title{
Missed pulmonary artery sarcoma requiring radical excision and pneumonectomy
}

Mohsyn Imran Malik, BSc, ${ }^{\mathrm{a}}$ Mohamad Rabbani, MD, CM, ${ }^{\mathrm{a}}$ Fadi Hage, MD, MPH, ${ }^{\mathrm{a}}$ Richard Inculet, MD, ${ }^{\mathrm{b}, \mathrm{c}}$ and Michael W. A. Chu, MD, MEd, ${ }^{\mathrm{a}}$ London, Ontario, Canada

From the Divisions of ${ }^{\mathrm{a}}$ Cardiac Surgery, ${ }^{\mathrm{b}}$ Thoracic Surgery, and ${ }^{\mathrm{c}}$ Oncology, Western University, London, Ontario, Canada.

Disclosures: M.W.C. has received Speakers' honoraria from Medtronic, Edwards Lifesciences, and Terumo Aortic, outside of the submitted work. All other authors report no conflicts of interest. All other authors reported no conflicts of interest.

The Journal policy requires editors and reviewers to disclose conflicts of interest and to decline handling or reviewing manuscripts for which they may have a conflict of interest. The editors and reviewers of this article have no conflicts of interest.

Received for publication April 15, 2021; accepted for publication Sept 27, 2021; available ahead of print Oct 12, 2021.

Address for reprints: Michael W. A. Chu, MD, MEd, London Health Sciences Centre, B6-106 University Hospital, 339 Windermere Rd, London, Ontario, Canada N6A 5A5 (E-mail: Michael.Chu@lhsc.on.ca).

JTCVS Techniques 2021;10:309-12

2666-2507

Copyright (c) 2021 The Author(s). Published by Elsevier Inc. on behalf of The American Association for Thoracic Surgery. This is an open access article under the CC BY-NC-ND license (http://creativecommons.org/licenses/bync-nd/4.0/).

https://doi.org/10.1016/j.xjtc.2021.09.055

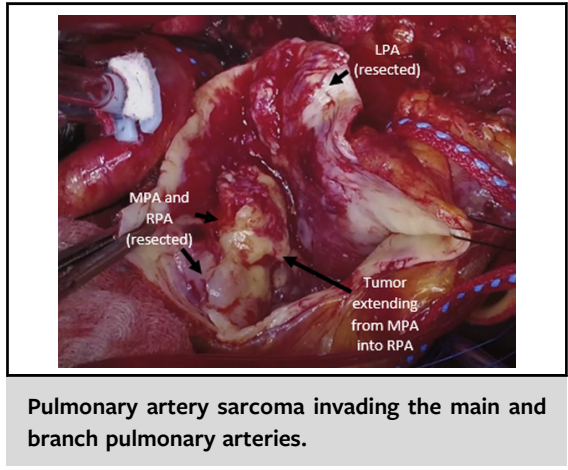

CENTRAL MESSAGE

A patient with a pulmonary artery sarcoma was misdiagnosed as having a pulmonary embolus, delaying definitive treatment with radical excision and pneumonectomy.

See Commentaries on pages 313 and 315.
A 64-year-old male patient was referred with 3-month history of progressive shortness of breath and nonexertional chest pain radiating to the back. The initial diagnosis on computed tomography (CT) scan was pulmonary embolus (Figure 1, $A-C$ ) and managed with anticoagulation therapy for 6 months. Follow-up CT demonstrated growth of the mass, prompting referral to thoracic surgery for further evaluation. A transthoracic echocardiogram demonstrated mild flow acceleration across the pulmonary valve and estimated pulmonary pressures of 55 to $60 \mathrm{~mm} \mathrm{Hg}$. Repeat CT scan identified an approximately $6-\mathrm{cm}$ mass filling the right pulmonary artery (RPA) to the hilum and extending down the main pulmonary artery (MPA), potentially involving the pulmonary valve (Figure $1, D-F$ ). The left pulmonary artery (LPA) appeared mostly spared. Multidisciplinary evaluation suggested concerns for pulmonary artery sarcoma (PAS), prompting consideration for urgent radical excision with homograft reconstruction and right pneumonectomy.

\section{TECHNIQUE}

A midline sternotomy was employed with central arterial and venous cannulation for cardiopulmonary bypass with cardioplegic arrest and cooling to $32^{\circ} \mathrm{C}$. Transection of the ascending aorta was necessary to provide optimal visualization for optimal tumor mobilization and resection. An anterior vertical incision was made in the MPA, which revealed complete tumor obstruction of the RPA orifice, extending distally down the LPA and proximally into the pulmonary valve commissure. An incision was made in the right ventricle outflow tract, and the pulmonary root was excised en-bloc with the tumor (Figure 2, A, Video 1). The MPA and pulmonary root was reflected superiorly, and dissection of the MPA off the posterior mediastinum was followed by resection of the MPA down the LPA to create a clear distal margin for the tumor. The LPA was circumferentially transected, and the resected LPA and MPA were reflected towards the RPA (Figure 2, B, Video 1). The LPA was reconstructed with a separate pulmonary branch homograft measuring approximately $3 \mathrm{~cm}$ using running 5-0 PROLENE sutures (Figure 2, C, Video 1). A 31-mm CryoLife SynerGraft pulmonary allograft was attached to the branch cuff using 2 running 5-0 PROLENE sutures (Figure 2, D, Video 1). The proximal end was 

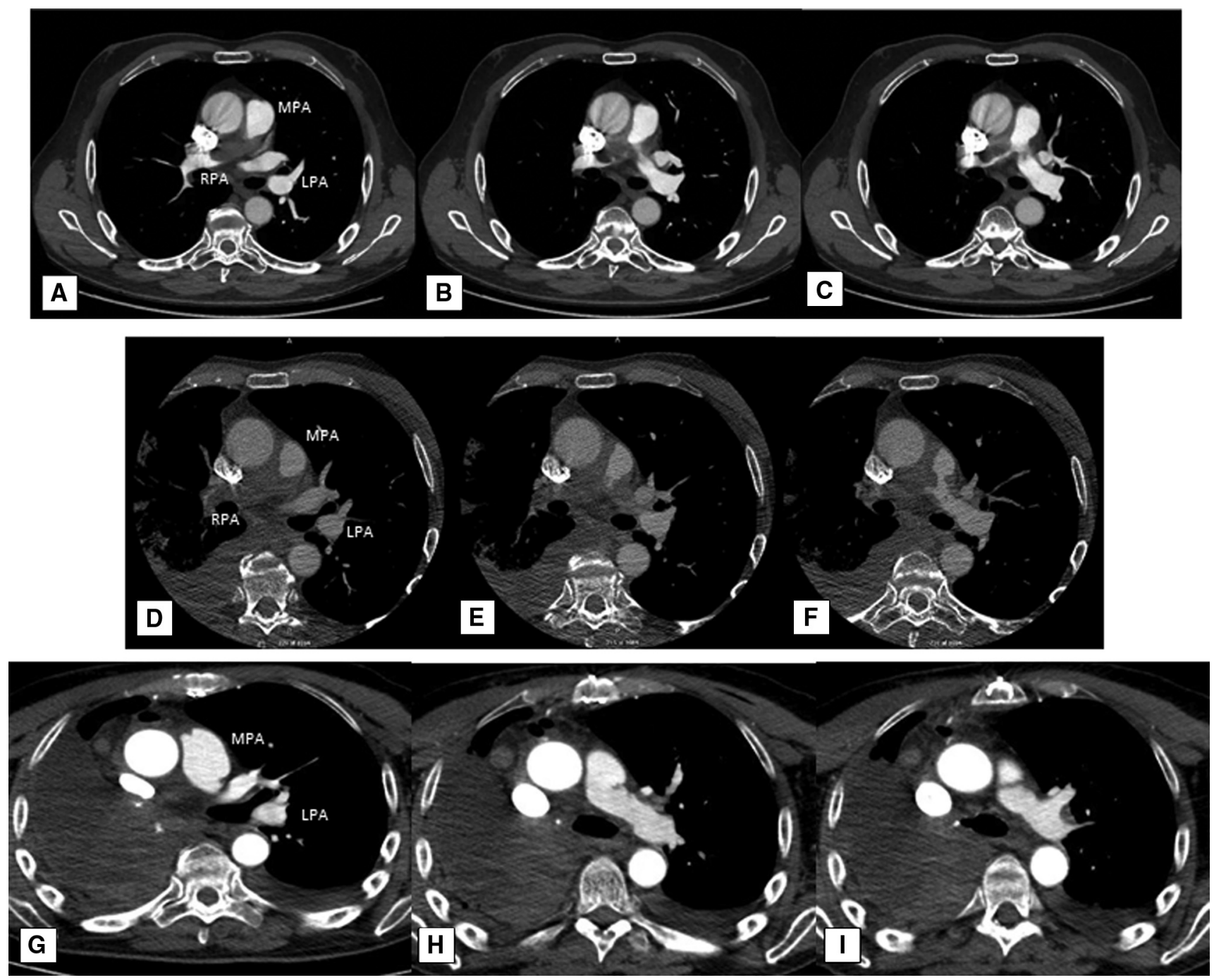

FIGURE 1. A-C, Initial CT during time of diagnosis of PE shows mass at bifurcation of the MPA with decreased filling of the RPA proximally. D-F, On preoperative cardiac CT, tumor can be seen invading into the MPA and causing full obstruction of the RPA. Extension into the LPA is difficult to determine. G-I, Postoperative cardiac CT demonstrates a patent reconstructed MPA and LPA. MPA, Main pulmonary artery; RPA, right pulmonary artery; LPA, left pulmonary artery.

reimplanted into the right ventricle outflow tract with running 4-0 PROLENE suture (Figure 2, E, Video 1). Reperfusate was administered, and the crossclamp was removed. The divided RPA was reflected into the right pleural space. Subsequent right pneumonectomy was amenable due to only minor chest wall adhesions, which were easily divided with electrocautery. The pulmonary veins and right mainstem bronchus were stapled and divided, and the right mainstem bronchial stump was oversewn. The right lung was removed with attached RPA, MPA, and portion of the LPA (Figure 2, $F$, Video 1). The patient was weaned from cardiopulmonary bypass. The right pleural space was filled with a bacitracin-povidone solution, and the pleural space was closed. Postoperative transesophageal echocardiogram demonstrated a wellfunctioning pulmonary homograft with only mild central pulmonary insufficiency. The patient was taken to the intensive care unit, where he was extubated on the first postoperative day and recovered on the ward without major complication before discharge on the seventh postoperative day. Pathology confirmed intimal sarcoma with a 5-mm resection margin and confirmed tumor extension through the right hilum into the lung parenchyma. At 6 months' follow-up, he was doing well, receiving adjuvant therapy with no signs of tumor recurrence. Repeat CT demonstrated a patent reconstructed MPA and LPA (Figure 1, G-I).

The institutional review board or equivalent ethics committee of Western University did not approve this study, as it was an anonymized retrospective individual case report without identifying data. The subject provided informed written consent for the publication of the study data. 


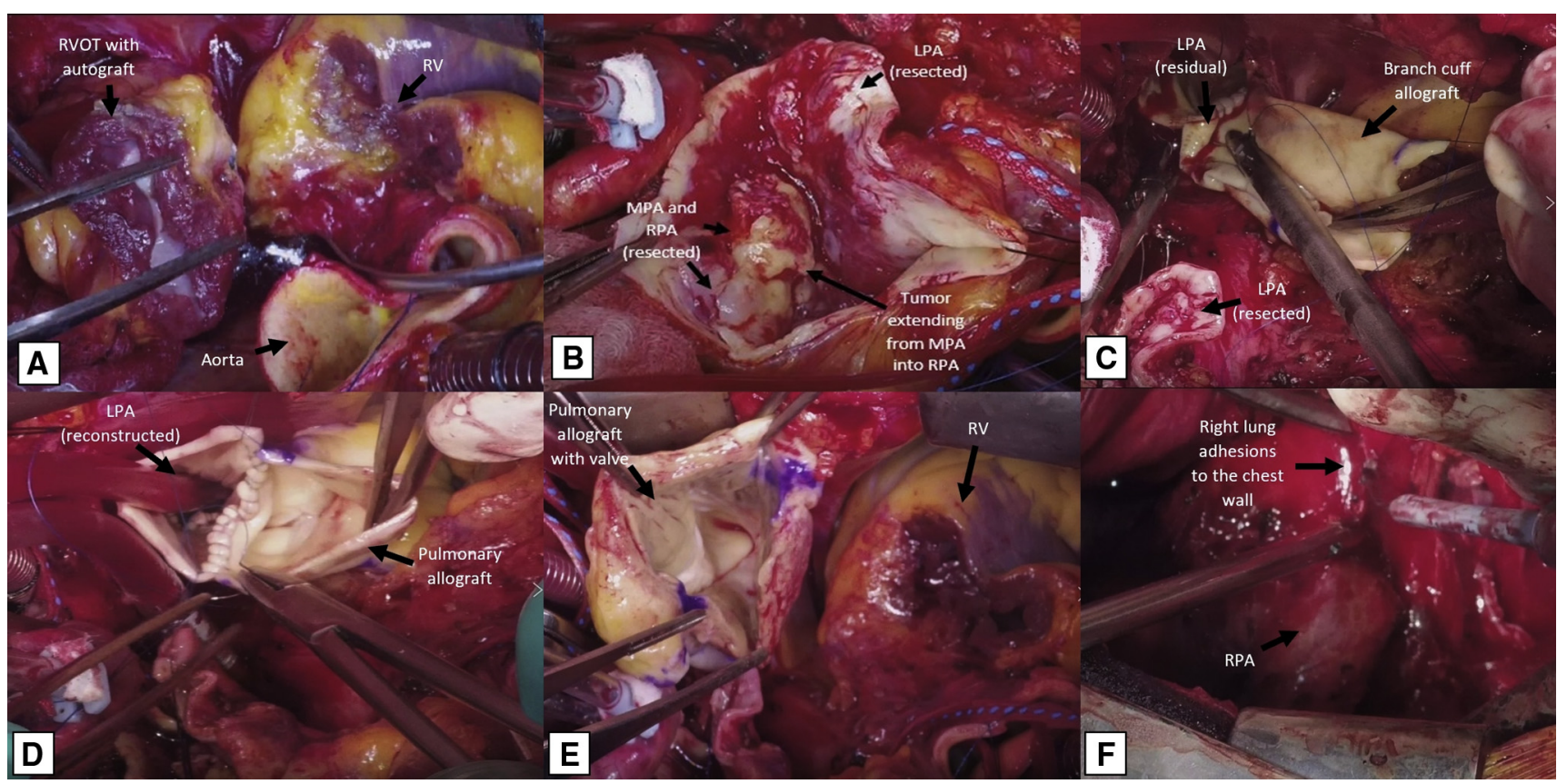

FIGURE 2. A, The RVOT and pulmonary root were harvested with cautery and scissors from the RV. B, Resected MPA, RPA, and about $50 \%$ LPA are reflected to demonstrate the tumor burden and obstruction of the RPA. C, A branch cuff allograft of about $3 \mathrm{~cm}$ was attached with sutures to the remaining native LPA D, Pulmonary autograft was attached with sutures to the newly reconstructed LPA branch cuff. E, Reconstruction was completed with attachment of the pulmonary autograft to the RV with sutures F, A view into the right pleural cavity showing lung adhesions to the chest wall, which were divided using cautery during pneumonectomy. $R V O T$, Right ventricular outflow tract; $R V$, right ventricle; $L P A$, left pulmonary artery; $M P A$, main pulmonary artery; $R P A$, right pulmonary artery.

\section{DISCUSSION}

Misdiagnosis of PAS for pulmonary embolus remains unfortunately common; however, distinguishing radiographic features include a low-attenuation filling defect occupying the entire luminal diameter, expansion of the pulmonary artery, extraluminal tumor spread, magnetic resonance imaging gadolinium update, and fluorodeoxyglucose avidity on fluorodeoxyglucose-positron emission tomography

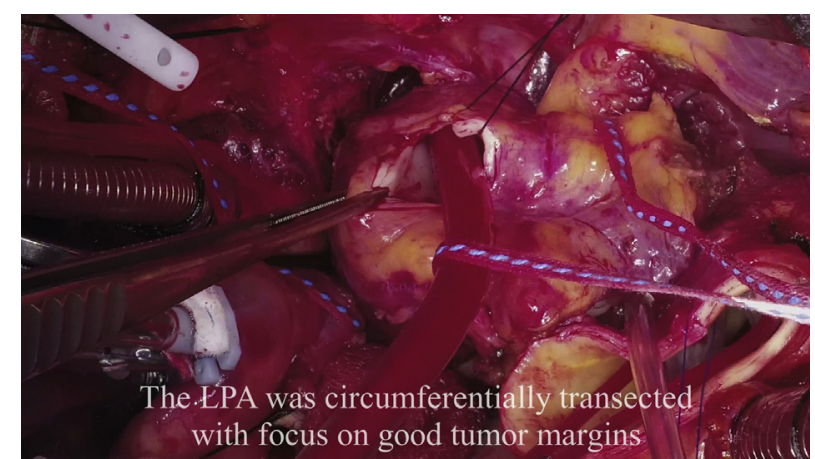

VIDEO 1. A step-by-step demonstration of our surgical approach for resection of bilateral PAS with pneumonectomy, and reconstruction of the pulmonary arteries and root. PAS, Pulmonary artery sarcoma. Video available at: https://www.jtcvs.org/article/S2666-2507(21)00689-1/ fulltext. scanning. ${ }^{1}$ Regardless, once sarcoma is suspected, early and complete surgical resection has been consistently demonstrated as the most important curative technique in PAS management. ${ }^{2-4}$ Despite this, bilateral PAS has been conventionally managed with palliative pulmonary endarterectomy, forgoing complete resection for symptomatic relief. ${ }^{2,3}$

With the goal of complete curative excision, we decided intraoperatively to continue with a radical resection and pneumonectomy. This required extensive planning, including ensuring homograft availability for reconstruction of the pulmonary artery. Cardioplegic arrest allowed for aortic transection, providing optimal exposure posterior to the aorta to determine tumor extension. Given partial extension into the LPA, we prioritized good margins distally toward the left lung and used an additional pulmonary allograft for reconstruction. Complete obstruction of the RPA and extension into the right lung necessitated a right pneumonectomy. Other options could have included debulking and palliation strategies, heart-lung transplantation, and, in some cases, pulmonary-preserving procedures.

Although this technique requires meticulous planning and surgical expertise, it offers a reasonable alternative to conventional management with greater survival benefit. 
Along with recent advancements in neoadjuvant therapies, ${ }^{5}$ the hope is this approach can build on past literature and lead to improved late survival in patients diagnosed with this rare but deadly disease.

\section{References}

1. Yi CA, Lee KS, Choe YH, Han D, Kwon OJ, Kim S. Computed tomography in pulmonary artery sarcoma: distinguishing features from pulmonary embolic disease. J Comput Assist Tomogr. 2004;28:34-9.
2. Song W, Deng L, Zhu J, Zheng S, Wang H, Song Y, et al. Surgical treatment of pulmonary artery sarcoma: a report of 17 cases. Pulm Cir. 2021;11: 2045894020986394.

3. von Ballmoos MC, Chan EY, Reardon MJ. Imaging and surgical treatment of primary pulmonary artery sarcoma. Int J Cardiovasc Imaging. 2019;35:1429-33.

4. Blackmon SH, Rice DC, Correa AM, Mehran R, Putnam JB, Smythe WR, et al. Management of primary pulmonary artery sarcomas. Ann Thorac Surg. 2009; 87:977-84.

5. Yin K, Luo R, Wei Y, Wang F, Zhang Y, Karlson KJ, et al. Survival outcomes in patients with primary cardiac sarcoma in the United States. J Thorac Cardiovasc Surg. 2021;162:107-15.e2. 